\title{
Approximation of fixed points for nonexpansive semigroups in Hilbert spaces
}

\author{
Yonghong Yao ${ }^{1}$, Jung Im Kang ${ }^{2 *}$, Yeol Je Cho ${ }^{3 *}$ and Yeong-Cheng Liou ${ }^{4}$
}

\author{
"Correspondence: \\ k9338sj@gmail.com; \\ yjcho@gsnu.ac.kr \\ ${ }^{2}$ National Institute for Mathematical \\ Sciences, KT Daeduk 2 Research \\ Center, 463-1 Jeonmin-dong, \\ Yusung-gu, Daejeon, 305-390, Korea \\ ${ }^{3}$ Department of Mathematics \\ Education and the RINS, \\ Gyeongsang National University, \\ Chinju, 660-701, Korea \\ Full list of author information is \\ available at the end of the article
}

\begin{abstract}
In this paper, we propose two new algorithms for finding a common fixed point of a nonexpansive semigroup in Hilbert spaces and prove some strong convergence theorems for nonexpansive semigroups. Our results improve and generalize the corresponding results given by Shimizu and Takahashi (J. Math. Anal. Appl. 211:71-83, 1997), Shioji and Takahashi (Nonlinear Anal. TMA 34:87-99, 1998), Lau et al. (Nonlinear Anal. TMA 67:1211-1225, 2007) and many others.
\end{abstract}

MSC: $47 \mathrm{H} 05 ; 47 \mathrm{H} 10 ; 47 \mathrm{H} 17$

Keywords: nonexpansive semigroup; common fixed point; algorithms; projection

\section{Introduction}

Let $H$ be a real Hilbert space with the inner product $\langle\cdot, \cdot\rangle$ and the norm $\|\cdot\|$. Let $C$ be a nonempty closed convex subset of $H$. A mapping $T: C \rightarrow C$ is said to be nonexpansive if

$$
\|T x-T y\| \leq\|x-y\|, \quad \forall x, y \in C .
$$

Recall that a family $S:=\{T(s)\}_{s \geq 0}$ of mappings of $C$ into itself is called a nonexpansive semigroup if it satisfies the following conditions:

(S1) $T(0) x=x$ for all $x \in C$

(S2) $T(s+t)=T(s) T(t)$ for all $s, t \geq 0$;

(S3) $\|T(s) x-T(s) y\| \leq\|x-y\|$ for all $x, y \in C$ and $s \geq 0$;

(S4) for each $x \in H, s \rightarrow T(s) x$ is continuous.

We denote by $\operatorname{Fix}(T(s))$ the set of fixed points of $T(s)$ and by $\operatorname{Fix}(S)$ the set of all common fixed points of $S$, i.e., $\operatorname{Fix}(S)=\bigcap_{s>0} \operatorname{Fix}(T(s)$ ). It is known that $\operatorname{Fix}(S)$ is closed and convex [1, Lemma 1].

Approximation of fixed points of nonexpansive mappings by a sequence of finite means has been considered by many authors; see, for instance, [1-27]. This work was originated with the beautiful work of Baillon [5] in 1975 (see also [6] and [7] for a generalization): If $C$ is a closed convex subset of a Hilbert space and $T$ is a nonexpansive mapping from $C$ into itself such that the set $\operatorname{Fix}(T)$ of fixed points of $T$ is nonempty, then for each $x \in C$, the Cesàro mean

$$
\frac{1}{n} \sum_{k=1}^{n} T^{k} x
$$

○ 2013 Yao et al.; licensee Springer. This is an Open Access article distributed under the terms of the Creative Commons Attribution License (http://creativecommons.org/licenses/by/2.0), which permits unrestricted use, distribution, and reproduction in any medium, provided the original work is properly cited. 
converges weakly to $x^{*} \in \operatorname{Fix}(T)$. In this case, if we put $x^{*}=P_{\mathrm{Fix}(T)} x$ for each $x \in C$, then $P_{\mathrm{Fix}(T)}$ is a nonexpansive retraction from $C$ onto $F(T)$. In [18], Takahashi proved the existence of such a retraction for an amenable semigroup of nonexpansive mappings on a Hilbert space. In [19], Rodé also found a sequence of means on a semigroup generalizing the Cesàro means and extended Baillon's theorem. In [28], Lau, Shioji and Takahashi extended Takahashi's result and Rode's result to a closed convex subset of a uniformly convex Banach space.

In the literature, a nonlinear ergodic theorem for nonexpansive semigroups has been considered by many authors (see [29-46]). Especially, Shioji and Takahashi [17] introduced an implicit iteration $\left\{x_{n}\right\}$ in a Hilbert space defined by

$$
x_{n}=\alpha_{n} x+\left(1-\alpha_{n}\right) \frac{1}{\lambda_{n}} \int_{0}^{\lambda_{n}} T(s) x_{n} d s, \quad \forall n \geq 0,
$$

where $\left\{\alpha_{n}\right\}$ is a sequence in $(0,1)$ and $\left\{\lambda_{n}\right\}$ is a sequence of positive real numbers divergent to $\infty$. Under certain restrictions on the sequence $\left\{\alpha_{n}\right\}$, Shioji and Takahashi [17] proved strong convergence of $\left\{x_{n}\right\}$ generated by (1.1) to a member of Fix $(T(s))$. In [16], Shimizu and Takahashi studied the strong convergence of the iterative sequence $\left\{x_{n}\right\}$ defined by

$$
x_{n+1}=\alpha_{n} x+\left(1-\alpha_{n}\right) \frac{1}{\lambda_{n}} \int_{0}^{\lambda_{n}} T(s) x_{n} d s, \quad \forall n \geq 0 .
$$

The corresponding viscosity approximations of (1.1) and (1.2) have been extended in [29]. Lau et al. [37] studied the iterative schemes of Browder and Halpern types for a nonexpansive semigroup $\{T(s)\}_{s \geq 0}$ on a compact convex subset $C$ of a smooth (and strictly convex) Banach space with respect to a sequence $\left\{\mu_{n}\right\}$ of strongly asymptotically invariant means defined on an appropriate invariant subspace of $l^{\infty}(S)$, the space of bounded real-valued functions on a semigroup $S$.

Motivated and inspired by the works in the literature, in this paper, we introduce two new algorithms for finding a common fixed point of a nonexpansive semigroup $\{T(s)\}_{s \geq 0}$ in Hilbert spaces and prove that both approaches converge strongly to a common fixed point of $\{T(s)\}_{s \geq 0}$.

\section{Preliminaries}

Let $C$ be a nonempty closed convex subset of a real Hilbert space $H$. The metric (or nearest point) projection from $H$ onto $C$ is the mapping $P_{C}: H \rightarrow C$ which assigns to each point $x \in C$ the unique point $P_{C} x \in C$ satisfying the property

$$
\left\|x-P_{C} x\right\|=\inf _{y \in C}\|x-y\|=: d(x, C) .
$$

It is well known that $P_{C}$ is a nonexpansive mapping and satisfies

$$
\left\langle x-y, P_{C} x-P_{C} y\right\rangle \geq\left\|P_{C} x-P_{C} y\right\|^{2}, \quad \forall x, y \in H .
$$

Moreover, $P_{C}$ is characterized by the following properties:

$$
\left\langle x-P_{C} x, y-P_{C} x\right\rangle \leq 0
$$


and

$$
\|x-y\|^{2} \geq\left\|x-P_{C} x\right\|^{2}+\left\|y-P_{C} x\right\|^{2}, \quad \forall x \in H, y \in C .
$$

We need the following lemmas for proving our main results.

Lemma 2.1 [16] Let $C$ be a nonempty bounded closed convex subset of a Hilbert space $H$ and $\{T(s)\}_{s \geq 0}$ be a nonexpansive semigroup on $C$. Then, for any $h \geq 0$,

$$
\lim _{t \rightarrow \infty} \sup _{x \in C}\left\|\frac{1}{t} \int_{0}^{t} T(s) x d s-T(h) \frac{1}{t} \int_{0}^{t} T(s) x d s\right\|=0 .
$$

Lemma 2.2 [8] Let $C$ be a closed convex subset of a real Hilbert space $H$ and $S: C \rightarrow C$ be a nonexpansive mapping. Then the mapping $I-S$ is demiclosed. That is, if $\left\{x_{n}\right\}$ is a sequence in $C$ such that $x_{n} \rightarrow x^{*}$ weakly and $(I-S) x_{n} \rightarrow y$ strongly, then $(I-S) x^{*}=y$.

Lemma 2.3 [13] Let $\left\{x_{n}\right\}$ and $\left\{y_{n}\right\}$ be bounded sequences in a Banach space $X$ and $\left\{\gamma_{n}\right\}$ be a sequence in $[0,1]$ with $0<\liminf _{n \rightarrow \infty} \beta_{n} \leq \limsup _{n \rightarrow \infty} \beta_{n}<1$. Suppose that

$$
x_{n+1}=\left(1-\gamma_{n}\right) x_{n}+\gamma_{n} y_{n}, \quad \forall n \geq 0,
$$

and

$$
\limsup _{n \rightarrow \infty}\left(\left\|y_{n}-y_{n-1}\right\|-\left\|x_{n}-x_{n-1}\right\|\right) \leq 0
$$

Then $\lim _{n \rightarrow \infty}\left\|y_{n}-x_{n}\right\|=0$.

Lemma 2.4 [12] Assume that $\left\{a_{n}\right\}$ is a sequence of nonnegative real numbers such that

$$
a_{n+1} \leq\left(1-\gamma_{n}\right) a_{n}+\delta_{n} \gamma_{n}, \quad \forall n \geq 1
$$

where $\left\{\gamma_{n}\right\}$ is a sequence in $(0,1)$ and $\left\{\delta_{n}\right\}$ is a sequence such that

(a) $\sum_{n=1}^{\infty} \gamma_{n}=\infty$;

(b) $\limsup _{n \rightarrow \infty} \delta_{n} \leq 0$ or $\sum_{n=1}^{\infty}\left|\delta_{n} \gamma_{n}\right|<\infty$.

Then $\lim _{n \rightarrow \infty} a_{n}=0$.

\section{Main results}

In this section, we show our main results.

Theorem 3.1 Let $C$ be a nonempty closed convex subset of a real Hilbert space H. Let $S=\{T(s)\}_{s \geq 0}: C \rightarrow C$ be a nonexpansive semigroup with $\operatorname{Fix}(S) \neq \emptyset$. Let $\left\{\gamma_{t}\right\}_{0<t<1}$ and $\left\{\lambda_{t}\right\}_{0<t<1}$ be two continuous nets of positive real numbers such that $\gamma_{t} \in(0,1), \lim _{t \rightarrow 0} \gamma_{t}=1$ and $\lim _{t \rightarrow 0} \lambda_{t}=+\infty$. Let $\left\{x_{t}\right\}$ be the net defined in the following implicit manner:

$$
x_{t}=P_{C}\left[t\left(\gamma_{t} x_{t}\right)+(1-t) \frac{1}{\lambda_{t}} \int_{0}^{\lambda_{t}} T(s) x_{t} d s\right], \quad \forall t \in(0,1) .
$$

Then, as $t \rightarrow 0+$, the net $\left\{x_{t}\right\}$ strongly converges to $x^{*} \in \operatorname{Fix}(S)$. 
Proof First, we note that the net $\left\{x_{t}\right\}$ defined by (3.1) is well defined. We define the mapping

$$
W x:=P_{C}\left[t\left(\gamma_{t} x\right)+(1-t) \frac{1}{\lambda_{t}} \int_{0}^{\lambda_{t}} T(s) x d s\right], \quad \forall t \in(0,1)
$$

It follows that

$$
\begin{aligned}
\|W x-W y\| & \leq\left\|t \gamma_{t}(x-y)+(1-t) \frac{1}{\lambda_{t}} \int_{0}^{\lambda_{t}}(T(s) x-T(s) y) d s\right\| \\
& \leq t \gamma_{t}\|x-y\|+(1-t)\left\|\frac{1}{\lambda_{t}} \int_{0}^{\lambda_{t}}(T(s) x-T(s) y) d s\right\| \\
& \leq t \gamma_{t}\|x-y\|+(1-t)\|x-y\| \\
& =\left[1-\left(1-\gamma_{t}\right) t\right]\|x-y\| .
\end{aligned}
$$

This implies that the mapping $W$ is a contraction and so it has a unique fixed point. Therefore, the net $\left\{x_{t}\right\}$ defined by (3.1) is well defined.

Take $p \in \operatorname{Fix}(S)$. By (3.1), we have

$$
\begin{aligned}
\left\|x_{t}-p\right\| & =\left\|P_{C}\left[t\left(\gamma_{t} x_{t}\right)+(1-t) \frac{1}{\lambda_{t}} \int_{0}^{\lambda_{t}} T(s) x_{t} d s\right]-p\right\| \\
& \leq\left\|t \gamma_{t}\left(x_{t}-p\right)-t\left(1-\gamma_{t}\right) p+(1-t)\left(\frac{1}{\lambda_{t}} \int_{0}^{\lambda_{t}} T(s) x_{t} d s-p\right)\right\| \\
& \leq t \gamma_{t}\left\|x_{t}-p\right\|+t\left(1-\gamma_{t}\right)\|p\|+(1-t) \frac{1}{\lambda_{t}} \int_{0}^{\lambda_{t}}\left\|T(s) x_{t}-T(s) p\right\| d s \\
& \leq t \gamma_{t}\left\|x_{t}-p\right\|+t\left(1-\gamma_{t}\right)\|p\|+(1-t)\left\|x_{t}-p\right\| \\
& =\left[1-\left(1-\gamma_{t}\right) t\right]\left\|x_{t}-p\right\|+t\left(1-\gamma_{t}\right)\|p\| .
\end{aligned}
$$

It follows that

$$
\left\|x_{t}-p\right\| \leq\|p\|
$$

which implies that the net $\left\{x_{t}\right\}$ is bounded. Set $R:=\|p\|$. It is clear that $\left\{x_{t}\right\} \subset B(p, R)$. Notice that

$$
\left\|\frac{1}{\lambda_{t}} \int_{0}^{\lambda_{t}} T(s) x_{t} d s-p\right\| \leq\left\|x_{t}-p\right\| \leq R
$$

Moreover, we observe that if $x \in B(p, R)$, then

$$
\|T(s) x-p\| \leq\|T(s) x-T(s) p\| \leq\|x-p\| \leq R
$$

i.e., $B(p, R)$ is $T(s)$-invariant for all $s$. Set $y_{t}=t\left(\gamma_{t} x_{t}\right)+(1-t) \frac{1}{\lambda_{t}} \int_{0}^{\lambda_{t}} T(s) x_{t} d s$. Then $x_{t}=$ $P_{C}\left[y_{t}\right]$. It follows that

$$
\begin{aligned}
& \left\|T(\tau) x_{t}-x_{t}\right\| \\
& \quad=\left\|P_{C}\left[T(\tau) x_{t}\right]-P_{C}\left[y_{t}\right]\right\|
\end{aligned}
$$




$$
\begin{aligned}
\leq & \left\|T(\tau) x_{t}-y_{t}\right\| \\
\leq & \left\|T(\tau) x_{t}-T(\tau) \frac{1}{\lambda_{t}} \int_{0}^{\lambda_{t}} T(s) x_{t} d s\right\| \\
& +\left\|T(\tau) \frac{1}{\lambda_{t}} \int_{0}^{\lambda_{t}} T(s) x_{t} d s-\frac{1}{\lambda_{t}} \int_{0}^{\lambda_{t}} T(s) x_{t} d s\right\|+\left\|\frac{1}{\lambda_{t}} \int_{0}^{\lambda_{t}} T(s) x_{t} d s-y_{t}\right\| \\
\leq & \left\|T(\tau) \frac{1}{\lambda_{t}} \int_{0}^{\lambda_{t}} T(s) x_{t} d s-\frac{1}{\lambda_{t}} \int_{0}^{\lambda_{t}} T(s) x_{t} d s\right\| \\
& +\left\|x_{t}-\frac{1}{\lambda_{t}} \int_{0}^{\lambda_{t}} T(s) x_{t} d s\right\|+t\left\|_{t} x_{t}-\frac{1}{\lambda_{t}} \int_{0}^{\lambda_{t}} T(s) x_{t} d s\right\| \\
\leq & \left\|T(\tau) \frac{1}{\lambda_{t}} \int_{0}^{\lambda_{t}} T(s) x_{t} d s-\frac{1}{\lambda_{t}} \int_{0}^{\lambda_{t}} T(s) x_{t} d s\right\|+2 t\left\|\gamma_{t} x_{t}-\frac{1}{\lambda_{t}} \int_{0}^{\lambda_{t}} T(s) x_{t} d s\right\| .
\end{aligned}
$$

By Lemma 2.1, we deduce that for all $0 \leq \tau<\infty$,

$$
\lim _{t \rightarrow 0}\left\|T(\tau) x_{t}-x_{t}\right\|=0
$$

Note that $x_{t}=P_{C}\left[y_{t}\right]$. By using the property of the metric projection (2.1), we have

$$
\begin{aligned}
\left\|x_{t}-p\right\|^{2}= & \left\langle x_{t}-y_{t}, x_{t}-p\right\rangle+\left\langle y_{t}-p, x_{t}-p\right\rangle \\
\leq & \left\langle y_{t}-p, x_{t}-p\right\rangle \\
= & t \gamma_{t}\left\langle x_{t}-p, x_{t}-p\right\rangle-t\left(1-\gamma_{t}\right)\left\langle p, x_{t}-p\right\rangle \\
& \quad+(1-t)\left\langle\frac{1}{\lambda_{t}} \int_{0}^{\lambda_{t}} T(s) x_{t} d s-p, x_{t}-p\right\rangle \\
\leq & {\left[1-\left(1-\gamma_{t}\right) t\right]\left\|x_{t}-p\right\|^{2}-t\left(1-\gamma_{t}\right)\left\langle p, x_{t}-p\right\rangle . }
\end{aligned}
$$

Therefore, we have

$$
\left\|x_{t}-p\right\|^{2} \leq\left\langle p, p-x_{t}\right\rangle, \quad \forall p \in \operatorname{Fix}(S)
$$

From this inequality, immediately it follows that $\omega_{w}\left(x_{t}\right)=\omega_{s}\left(x_{t}\right)$, where $\omega_{w}\left(x_{t}\right)$ and $\omega_{s}\left(x_{t}\right)$ denote the sets of weak and strong cluster points of $\left\{x_{t}\right\}$, respectively.

Let $\left\{t_{n}\right\} \subset(0,1)$ be a sequence such that $t_{n} \rightarrow 0$ as $n \rightarrow \infty$. Put $x_{n}:=x_{t_{n}}, y_{n}:=y_{t_{n}}$ and $\lambda_{n}:=$ $\lambda_{t_{n}}$. Since $\left\{x_{n}\right\}$ is bounded, without loss of generality, we may assume that the sequence $\left\{x_{n}\right\}$ converges weakly to a point $x^{*} \in C$. Also, $y_{n} \rightarrow x^{*}$ weakly. Noticing (3.2), we can use Lemma 2.2 to get $x^{*} \in \operatorname{Fix}(S)$. From (3.3), we have

$$
\left\|x_{n}-p\right\|^{2} \leq\left\langle p, p-x_{n}\right\rangle, \quad \forall p \in \operatorname{Fix}(S)
$$

In particular, if we substitute $x^{*}$ for $p$ in (3.4), then we have

$$
\left\|x_{n}-x^{*}\right\|^{2} \leq\left\langle x^{*}, x^{*}-x_{n}\right\rangle
$$

However, $x_{n} \rightarrow x^{*}$. This together with (3.5) guarantees that $x_{n} \rightarrow x^{*}$ and so the net $\left\{x_{t}\right\}$ is relatively compact, as $t \rightarrow 0^{+}$, in the norm topology. 
Now, in (3.4), taking $n \rightarrow \infty$, we get

$$
\left\|x^{*}-p\right\|^{2} \leq\left\langle p, p-x^{*}\right\rangle, \quad \forall p \in \operatorname{Fix}(S)
$$

This is equivalent to the following:

$$
0 \leq\left\langle x^{*}, p-x^{*}\right\rangle, \quad \forall p \in \operatorname{Fix}(S)
$$

Therefore, $x^{*}=P_{\operatorname{Fix}(T)}(0)$, which is obviously unique. This is sufficient to conclude that the entire net $\left\{x_{t}\right\}$ converges in norm to $x^{*}$. This completes the proof.

Remark 3.2 It is known that the algorithm

$$
x_{t}=P_{C}\left[t x_{t}+(1-t) \frac{1}{\lambda_{t}} \int_{0}^{\lambda_{t}} T(s) x_{t} d s\right], \quad \forall t \in(0,1),
$$

has only weak convergence. However, our similar algorithm (3.1) (with $\gamma_{t} \rightarrow 1$ ) has strong convergence.

Next, we introduce an explicit algorithm for the nonexpansive semigroup $S=\{T(s)\}_{s \geq 0}$ : $C \rightarrow C$ and prove the strong convergence theorems of this algorithm.

Theorem 3.3 Let $C$ be a nonempty closed convex subset of a real Hilbert space $H$. Let $S=$ $\{T(s)\}_{s \geq 0}: C \rightarrow C$ be a nonexpansive semigroup with $\operatorname{Fix}(S) \neq \emptyset$. Let $\left\{x_{n}\right\}$ be the sequence generated iteratively by the following explicit algorithm:

$$
x_{n+1}=\left(1-\beta_{n}\right) x_{n}+\beta_{n} P_{C}\left[\alpha_{n}\left(\gamma_{n} x_{n}\right)+\left(1-\alpha_{n}\right) \frac{1}{\lambda_{n}} \int_{0}^{\lambda_{n}} T(s) x_{n} d s\right], \quad \forall n \geq 0 \text {, }
$$

where $\left\{\alpha_{n}\right\},\left\{\beta_{n}\right\}$ and $\left\{\gamma_{n}\right\}$ are sequences of real numbers in $[0,1]$ and $\left\{\lambda_{n}\right\}$ is a sequence of positive real numbers. Suppose that the following conditions are satisfied:

(i) $\lim _{n \rightarrow \infty} \alpha_{n}=0, \sum_{n=0}^{\infty} \alpha_{n}=\infty$ and $\lim _{n \rightarrow \infty} \gamma_{n}=1$;

(ii) $0<\liminf _{n \rightarrow \infty} \beta_{n} \leq \limsup _{n \rightarrow \infty} \beta_{n}<1$;

(iii) $\lim _{n \rightarrow \infty} \lambda_{n}=\infty$ and $\lim _{n \rightarrow \infty} \frac{\lambda_{n-1}}{\lambda_{n}}=1$.

Then the sequence $\left\{x_{n}\right\}$ generated by (3.6) strongly converges to a point $x^{*} \in \operatorname{Fix}(S)$.

Proof Take $p \in \operatorname{Fix}(S)$. From (3.6), we have

$$
\begin{aligned}
& \left\|x_{n+1}-p\right\| \\
& =\left\|\left(1-\beta_{n}\right) x_{n}+\beta_{n} P_{C}\left[\alpha_{n}\left(\gamma_{n} x_{n}\right)+\left(1-\alpha_{n}\right) \frac{1}{\lambda_{n}} \int_{0}^{\lambda_{n}} T(s) x_{n} d s\right]-p\right\| \\
& \leq\left(1-\beta_{n}\right)\left\|x_{n}-p\right\|+\beta_{n}\left\|P_{C}\left[\alpha_{n}\left(\gamma_{n} x_{n}\right)+\left(1-\alpha_{n}\right) \frac{1}{\lambda_{n}} \int_{0}^{\lambda_{n}} T(s) x_{n} d s\right]-p\right\| \\
& \leq\left(1-\beta_{n}\right)\left\|x_{n}-p\right\|+\beta_{n} \| \alpha_{n} \gamma_{n}\left(x_{n}-p\right)-\alpha_{n}\left(1-\gamma_{n}\right) p \\
& \quad+\left(1-\alpha_{n}\right)\left(\frac{1}{\lambda_{n}} \int_{0}^{\lambda_{n}} T(s) x_{n} d s-p\right) \|
\end{aligned}
$$




$$
\begin{aligned}
& \leq\left(1-\beta_{n}\right)\left\|x_{n}-p\right\|+\beta_{n}\left(\alpha_{n} \gamma_{n}\left\|x_{n}-p\right\|+\alpha_{n}\left(1-\gamma_{n}\right)\|p\|\right. \\
& \left.\quad+\left(1-\alpha_{n}\right) \frac{1}{\lambda_{n}} \int_{0}^{\lambda_{n}}\left\|T(s) x_{n}-T(s) p\right\| d s\right) \\
& \leq\left(1-\beta_{n}\right)\left\|x_{n}-p\right\|+\beta_{n}\left(\alpha_{n} \gamma_{n}\left\|x_{n}-p\right\|+\alpha_{n}\left(1-\gamma_{n}\right)\|p\|+\left(1-\alpha_{n}\right)\left\|x_{n}-p\right\|\right) \\
& =\left[1-\left(1-\gamma_{n}\right) \alpha_{n} \beta_{n}\right]\left\|x_{n}-p\right\|+\left(1-\gamma_{n}\right) \alpha_{n} \beta_{n}\|p\| .
\end{aligned}
$$

It follows that, by induction,

$$
\left\|x_{n}-p\right\| \leq \max \left\{\left\|x_{0}-p\right\|,\|p\|\right\}
$$

Set $y_{n}=P_{C}\left[\alpha_{n}\left(\gamma_{n} x_{n}\right)+\left(1-\alpha_{n}\right) z_{n}\right]$ for all $n \geq 0$, where $z_{n}=\frac{1}{\lambda_{n}} \int_{0}^{\lambda_{n}} T(s) x_{n} d s$. We have

$$
\begin{aligned}
& \left\|y_{n}-y_{n-1}\right\| \\
& =\left\|P_{C}\left[\alpha_{n}\left(\gamma_{n} x_{n}\right)+\left(1-\alpha_{n}\right) z_{n}\right]-P_{C}\left[\alpha_{n-1}\left(\gamma_{n-1} x_{n-1}\right)-\left(1-\alpha_{n-1}\right) z_{n-1}\right]\right\| \\
& \leq\left\|\alpha_{n}\left(\gamma_{n} x_{n}\right)+\left(1-\alpha_{n}\right) z_{n}-\alpha_{n-1}\left(\gamma_{n-1} x_{n-1}\right)-\left(1-\alpha_{n-1}\right) z_{n-1}\right\| \\
& =\| \alpha_{n} \gamma_{n}\left(x_{n}-x_{n-1}\right)+\left(\alpha_{n} \gamma_{n}-\alpha_{n-1} \gamma_{n-1}\right) x_{n-1} \\
& \quad+\left(1-\alpha_{n}\right)\left(z_{n}-z_{n-1}\right)+\left(\alpha_{n-1}-\alpha_{n}\right) z_{n-1} \| \\
& \leq \\
& \quad \alpha_{n} \gamma_{n}\left\|x_{n}-x_{n-1}\right\|+\left|\alpha_{n} \gamma_{n}-\alpha_{n-1} \gamma_{n-1}\right|\left\|x_{n-1}\right\|+\left|\alpha_{n-1}-\alpha_{n}\right|\left\|z_{n-1}\right\| \\
& \quad+\left(1-\alpha_{n}\right)\left\|z_{n}-z_{n-1}\right\|
\end{aligned}
$$

and

$$
\begin{aligned}
\| z_{n}- & z_{n-1} \| \\
= & \| \frac{1}{\lambda_{n}} \int_{0}^{\lambda_{n}}\left[T(s) x_{n}-T(s) x_{n-1}\right] d s+\left(\frac{1}{\lambda_{n}}-\frac{1}{\lambda_{n-1}}\right) \int_{0}^{\lambda_{n-1}} T(s) x_{n-1} d s \\
& +\frac{1}{\lambda_{n}} \int_{\lambda_{n-1}}^{\lambda_{n}} T(s) x_{n-1} d s \| \\
\leq & \frac{1}{\lambda_{n}} \int_{0}^{\lambda_{n}}\left\|T(s) x_{n}-T(s) x_{n-1}\right\| d s+\frac{1}{\lambda_{n}}\left\|\int_{\lambda_{n-1}}^{\lambda_{n}}\left[T(s) x_{n-1}-T(s) p\right] d s\right\| \\
& +\left|\frac{1}{\lambda_{n}}-\frac{1}{\lambda_{n-1}}\right| \int_{0}^{\lambda_{n-1}}\left\|T(s) x_{n-1}-T(s) p\right\| d s \\
\leq & \left\|x_{n}-x_{n-1}\right\|+\frac{2\left|\lambda_{n}-\lambda_{n-1}\right|}{\lambda_{n}}\left\|x_{n-1}-p\right\| .
\end{aligned}
$$

Therefore, we have

$$
\begin{aligned}
& \left\|y_{n}-y_{n-1}\right\| \\
& \leq \alpha_{n} \gamma_{n}\left\|x_{n}-x_{n-1}\right\|+\left|\alpha_{n} \gamma_{n}-\alpha_{n-1} \gamma_{n-1}\right|\left\|x_{n-1}\right\|+\left|\alpha_{n-1}-\alpha_{n}\right|\left\|z_{n-1}\right\| \\
& \quad+\left(1-\alpha_{n}\right)\left\|x_{n}-x_{n-1}\right\|+\frac{2\left|\lambda_{n}-\lambda_{n-1}\right|}{\lambda_{n}}\left\|x_{n-1}-p\right\| \\
& \quad \leq\left[1-\left(1-\gamma_{n}\right) \alpha_{n}\right]\left\|x_{n}-x_{n-1}\right\|+M\left(\left|\alpha_{n} \gamma_{n}-\alpha_{n-1} \gamma_{n-1}\right|+\left|\alpha_{n}-\alpha_{n-1}\right|+\frac{\left|\lambda_{n}-\lambda_{n-1}\right|}{\lambda_{n}}\right)
\end{aligned}
$$


where $M>0$ is a constant such that

$$
\sup _{n \geq 1}\left\{\left\|x_{n-1}\right\|,\left\|z_{n-1}\right\|, 2\left\|x_{n-1}-p\right\|\right\} \leq M
$$

Hence we get

$$
\limsup _{n \rightarrow \infty}\left(\left\|y_{n}-y_{n-1}\right\|-\left\|x_{n}-x_{n-1}\right\|\right) \leq 0 .
$$

This together with Lemma 2.3 implies that

$$
\lim _{n \rightarrow \infty}\left\|y_{n}-x_{n}\right\|=0
$$

Therefore, it follows that

$$
\lim _{n \rightarrow \infty}\left\|x_{n+1}-x_{n}\right\|=\lim _{n \rightarrow \infty} \beta_{n}\left\|y_{n}-x_{n}\right\|=0 .
$$

Note that

$$
\begin{aligned}
\left\|T(\tau) x_{n}-x_{n}\right\| \leq & \left\|T(\tau) x_{n}-T(\tau) \frac{1}{\lambda_{n}} \int_{0}^{\lambda_{n}} T(s) x_{n} d s\right\| \\
& +\left\|T(\tau) \frac{1}{\lambda_{n}} \int_{0}^{\lambda_{n}} T(s) x_{n} d s-\frac{1}{\lambda_{n}} \int_{0}^{\lambda_{n}} T(s) x_{n} d s\right\| \\
& +\left\|\frac{1}{\lambda_{n}} \int_{0}^{\lambda_{n}} T(s) x_{n} d s-x_{n}\right\| \\
\leq & \left\|T(\tau) \frac{1}{\lambda_{n}} \int_{0}^{\lambda_{n}} T(s) x_{n} d s-\frac{1}{\lambda_{n}} \int_{0}^{\lambda_{n}} T(s) x_{n} d s\right\| \\
& +2\left\|x_{n}-\frac{1}{\lambda_{n}} \int_{0}^{\lambda_{n}} T(s) x_{n} d s\right\| .
\end{aligned}
$$

From (3.6), we have

$$
\begin{aligned}
& \left\|x_{n}-\frac{1}{\lambda_{n}} \int_{0}^{\lambda_{n}} T(s) x_{n} d s\right\| \\
& \leq\left\|x_{n}-x_{n+1}\right\|+\left\|x_{n+1}-\frac{1}{\lambda_{n}} \int_{0}^{\lambda_{n}} T(s) x_{n} d s\right\| \\
& \leq\left\|x_{n}-x_{n+1}\right\|+\left(1-\beta_{n}\right)\left\|x_{n}-\frac{1}{\lambda_{n}} \int_{0}^{\lambda_{n}} T(s) x_{n} d s\right\| \\
& \quad+\alpha_{n} \gamma_{n}\left\|x_{n}-\frac{1}{\lambda_{n}} \int_{0}^{\lambda_{n}} T(s) x_{n} d s\right\|+\alpha_{n}\left(1-\gamma_{n}\right)\left\|\frac{1}{\lambda_{n}} \int_{0}^{\lambda_{n}} T(s) x_{n} d s\right\| .
\end{aligned}
$$

It follows that

$$
\begin{aligned}
& \left\|x_{n}-\frac{1}{\lambda_{n}} \int_{0}^{\lambda_{n}} T(s) x_{n} d s\right\| \\
& \quad \leq \frac{1}{\beta_{n}-\alpha_{n} \gamma_{n}}\left[\left\|x_{n}-x_{n+1}\right\|+\alpha_{n}\left(1-\gamma_{n}\right)\left\|\frac{1}{\lambda_{n}} \int_{0}^{\lambda_{n}} T(s) x_{n} d s\right\|\right] \rightarrow 0 .
\end{aligned}
$$


From (3.7), (3.8) and Lemma 2.1, we have

$$
\lim _{n \rightarrow \infty}\left\|T(\tau) x_{n}-x_{n}\right\|=0, \quad \forall \tau \geq 0
$$

Notice that $\left\{x_{n}\right\}$ is a bounded sequence and $\tilde{x}$ is a weak limit of $\left\{x_{n}\right\}$. Putting $x^{*}=P_{\operatorname{Fix}(S)}(0)$. Then there exists a positive number $R$ such that $B\left(x^{*}, R\right)$ contains $\left\{x_{n}\right\}$. Moreover, $B\left(x^{*}, R\right)$ is $T(s)$-invariant for all $s \geq 0$ and so, without loss of generality, we can assume that $\{T(s)\}_{s \geq 0}$ is a nonexpansive semigroup on $B\left(x^{*}, R\right)$. By the demiclosedness principle (Lemma 2.2) and (3.9), we have $\tilde{x} \in \operatorname{Fix}(S)$ and hence

$$
\limsup _{n \rightarrow \infty}\left\langle x^{*}, x_{n+1}-x^{*}\right\rangle=\lim _{n \rightarrow \infty}\left\langle x^{*}, \tilde{x}-x^{*}\right\rangle \leq 0
$$

Finally, we prove that $x_{n} \rightarrow x^{*}$. Set $u_{n}=\alpha_{n}\left(\gamma_{n} x_{n}\right)+\left(1-\alpha_{n}\right) \frac{1}{\lambda_{n}} \int_{0}^{\lambda_{n}} T(s) x_{n} d s$. It follows that $y_{n}=P_{C}\left[u_{n}\right]$ for all $n \geq 0$. By using the property of the metric projection (2.1), we have

$$
\left\langle y_{n}-u_{n}, y_{n}-x^{*}\right\rangle \leq 0
$$

and so

$$
\begin{aligned}
\left\|y_{n}-x^{*}\right\|^{2}= & \left\langle y_{n}-x^{*}, y_{n}-x^{*}\right\rangle \\
= & \left\langle y_{n}-u_{n}, y_{n}-x^{*}\right\rangle+\left\langle u_{n}-x^{*}, y_{n}-x^{*}\right\rangle \\
\leq & \left\langle u_{n}-x^{*}, y_{n}-x^{*}\right\rangle \\
= & \alpha_{n} \gamma_{n}\left\langle x_{n}-x^{*}, y_{n}-x^{*}\right\rangle-\alpha_{n}\left(1-\gamma_{n}\right)\left\langle x^{*}, y_{n}-x^{*}\right\rangle \\
& +\left(1-\alpha_{n}\right)\left\langle z_{n}-x^{*}, y_{n}-x^{*}\right\rangle \\
\leq & \alpha_{n} \gamma_{n}\left\|x_{n}-x^{*}\right\|\left\|y_{n}-x^{*}\right\|-\alpha_{n}\left(1-\gamma_{n}\right)\left\langle x^{*}, y_{n}-x^{*}\right\rangle \\
& +\left(1-\alpha_{n}\right)\left\|z_{n}-x^{*}\right\|\left\|y_{n}-x^{*}\right\| \\
\leq & {\left[1-\left(1-\gamma_{n}\right) \alpha_{n}\right]\left\|x_{n}-x^{* *}\right\|\left\|y_{n}-x^{*}\right\|-\alpha_{n}\left(1-\gamma_{n}\right)\left\langle x^{*}, y_{n}-x^{*}\right\rangle } \\
\leq & \frac{1-\left(1-\gamma_{n}\right) \alpha_{n}}{2}\left\|x_{n}-x^{*}\right\|^{2}+\frac{1}{2}\left\|y_{n}-x^{*}\right\|-\alpha_{n}\left(1-\gamma_{n}\right)\left\langle x^{*}, y_{n}-x^{*}\right\rangle,
\end{aligned}
$$

that is,

$$
\left\|y_{n}-x^{*}\right\|^{2} \leq\left[1-\left(1-\gamma_{n}\right) \alpha_{n}\right]\left\|x_{n}-x^{*}\right\|^{2}-2 \alpha_{n}\left(1-\gamma_{n}\right)\left\langle x^{*}, y_{n}-x^{*}\right\rangle
$$

By the convexity of the norm, we have

$$
\begin{aligned}
\left\|x_{n+1}-x^{*}\right\|^{2} & \leq\left(1-\beta_{n}\right)\left\|x_{n}-x^{*}\right\|^{2}+\beta_{n}\left\|y_{n}-x^{*}\right\|^{2} \\
& \leq\left[1-\left(1-\gamma_{n}\right) \alpha_{n} \beta_{n}\right]\left\|x_{n}-x^{*}\right\|^{2}-2\left(1-\gamma_{n}\right) \alpha_{n} \beta_{n}\left\langle x^{*}, y_{n}-x^{*}\right\rangle .
\end{aligned}
$$

Hence all the conditions of Lemma 2.4 are satisfied. Therefore, we immediately deduce that $x_{n} \rightarrow x^{*}$. This completes the proof.

In Theorem 3.3, if we put $\beta_{n}=1$ for each $n \geq 1$, we have the following corollary. 
Corollary 3.4 Let $C$ be a nonempty closed convex subset of a real Hilbert space H. Let $S=\{T(s)\}_{s \geq 0}: C \rightarrow C$ be a nonexpansive semigroup with $\operatorname{Fix}(S) \neq \emptyset$. Let the sequence $\left\{x_{n}\right\}$ be generated iteratively by the following explicit algorithm:

$$
x_{n+1}=P_{C}\left[\alpha_{n}\left(\gamma_{n} x_{n}\right)+\left(1-\alpha_{n}\right) \frac{1}{\lambda_{n}} \int_{0}^{\lambda_{n}} T(s) x_{n} d s\right], \quad \forall n \geq 0,
$$

where $\left\{\alpha_{n}\right\},\left\{\beta_{n}\right\}$ and $\left\{\gamma_{n}\right\}$ are sequences of real numbers in $[0,1]$ and $\left\{\lambda_{n}\right\}$ is a sequence of positive real numbers. Suppose that the following conditions are satisfied:

(i) $\lim _{n \rightarrow \infty} \alpha_{n}=0, \sum_{n=0}^{\infty} \alpha_{n}=\infty$ and $\lim _{n \rightarrow \infty} \gamma_{n}=1$;

(ii) $\lim _{n \rightarrow \infty} \lambda_{n}=\infty$ and $\lim _{n \rightarrow \infty} \frac{\lambda_{n-1}}{\lambda_{n}}=1$.

Then the sequence $\left\{x_{n}\right\}$ generated by (3.10) strongly converges to a point $x^{*} \in \operatorname{Fix}(S)$.

Remark 3.5 It is known that the algorithm

$$
x_{n+1}=\left(1-\beta_{n}\right) x_{n}+\beta_{n} P_{C}\left[\alpha_{n} x_{n}+\left(1-\alpha_{n}\right) \frac{1}{\lambda_{n}} \int_{0}^{\lambda_{n}} T(s) x_{n} d s\right], \quad \forall n \geq 0,
$$

has only weak convergence. However, our similar algorithm (3.6) (with $\gamma_{n} \rightarrow 1$ ) has strong convergence.

\section{Competing interests}

The authors declare that they have no competing interests.

\section{Authors' contributions}

All authors carried out the proof. All authors conceived of the study, and participated in its design and coordination. All authors read and approved the final manuscript.

\section{Author details}

${ }^{1}$ Department of Mathematics, Tianjin Polytechnic University, Tianjin, 300387, China. ${ }^{2}$ National Institute for Mathematical Sciences, KT Daeduk 2 Research Center, 463-1 Jeonmin-dong, Yusung-gu, Daejeon, 305-390, Korea. ${ }^{3}$ Department of Mathematics Education and the RINS, Gyeongsang National University, Chinju, 660-701, Korea. ${ }^{4}$ Department of Information Management, Cheng Shiu University, Kaohsiung, 833, Taiwan.

\section{Acknowledgements}

The third author was supported by the Basic Science Research Program through the National Research Foundation of Korea (NRF) funded by the Ministry of Education, Science and Technology (Grant Number: 2012-0008170).

\section{Received: 30 July 2012 Accepted: 30 January 2013 Published: 14 February 2013}

\section{References}

1. Browder, FE: Convergence of approximation to fixed points of nonexpansive nonlinear mappings in Hilbert spaces. Arch. Ration. Mech. Anal. 24, 82-90 (1967)

2. Browder, FE: Convergence theorems for sequences of nonlinear operators in Banach spaces. Math. Z. 100, 201-225 (1967)

3. Halpern, B: Fixed points of nonexpansive maps. Bull. Am. Math. Soc. 73, 957-961 (1967)

4. Lions, PL: Approximation de points fixes de contractions. C. R. Acad. Sci. Paris Sér. A-B 284, 1357-1359 (1977)

5. Baillon, JB: Un théoréme de type ergodique pour les contractions non linéaires dans un espace de Hilbert. C. R. Acad. Sci. Paris Sér. A-B 280, 1511-1514 (1975)

6. Brézis, H, Browder, FE: Nonlinear ergodic theorems. Bull. Am. Math. Soc. 82, 959-961 (1976)

7. Brézis, H, Browder, FE: Remarks on nonlinear ergodic theory. Adv. Math. 25, 165-177 (1977)

8. Geobel, K, Kirk, WA: Topics in Metric Fixed Point Theory. Cambridge Studies in Advanced Mathematics, vol. 28. Cambridge University Press, Cambridge (1990)

9. Marino, G, Xu, HK: A general iterative method for nonexpansive mappings in Hilbert spaces. J. Math. Anal. Appl. 318 43-52 (2006)

10. Moudafi, A: Viscosity approximation methods for fixed-points problems. J. Math. Anal. Appl. 241, 46-55 (2000)

11. Chang, SS: Viscosity approximation methods for a finite family of nonexpansive mappings in Banach spaces. J. Math. Anal. Appl. 323, 1402-1416 (2006)

12. $\mathrm{Xu}, \mathrm{HK}$ : Iterative algorithms for nonlinear operators. J. Lond. Math. Soc. 66, 240-256 (2002) 
13. Suzuki, T: Strong convergence theorems for infinite families of nonexpansive mappings in general Banach spaces. Fixed Point Theory Appl. 2005, 103-123 (2005)

14. Mainge, PE: Approximation methods for common fixed points of nonexpansive mappings in Hilbert spaces. J. Math. Anal. Appl. 325, 469-479 (2007)

15. Shioji, N, Takahashi, W: Strong convergence of approximated sequences for nonexpansive mappings in Banach spaces. Proc. Am. Math. Soc. 125, 3641-3645 (1997)

16. Shimizu, T, Takahashi, W: Strong convergence to common fixed points of families of nonexpansive mappings. J. Math. Anal. Appl. 211,71-83 (1997)

17. Shioji, N, Takahashi, W: Strong convergence theorems for asymptotically nonexpansive mappings in Hilbert spaces. Nonlinear Anal. TMA 34, 87-99 (1998)

18. Takahashi, W: A nonlinear ergodic theorem for an amenable semigroup of nonexpansive mappings in a Hilbert space. Proc. Am. Math. Soc. 81, 253-256 (1981)

19. Rodé, G: An ergodic theorem for semigroups of nonexpansive mappings in a Hilbert space. J. Math. Anal. Appl. 85, 172-178 (1982)

20. Zeng, LC, Yao, JC: Implicit iteration scheme with perturbed mapping for common fixed points of a finite family of nonexpansive mappings. Nonlinear Anal. TMA 64, 2507-2515 (2006)

21. Yao, Y, Chen, R, Liou, YC: A unified implicit algorithm for solving the triple-hierarchical constrained optimization problem. Math. Comput. Model. 55, 1506-1515 (2012)

22. Yao, Y, Shahzad, N: Strong convergence of a proximal point algorithm with general errors. Optim. Lett. (2012). doi:10.1007/s11590-011-0286-2

23. Yao, Y, Shahzad, N: New methods with perturbations for non-expansive mappings in Hilbert spaces. Fixed Point Theory Appl. 2011, 79 (2011)

24. Yao, Y, Yao, JC: On modified iterative method for nonexpansive mappings and monotone mappings. Appl. Math. Comput. 186, 1551-1558 (2007)

25. Plubtieng, S, Wangkeeree, $\mathrm{R}$ : Strong convergence of modified Mann iterations for a countable family of nonexpansive mappings. Nonlinear Anal. TMA 70, 3110-3118 (2009)

26. Cho, YJ, Qin, X: Convergence of a general iterative method for nonexpansive mappings in Hilbert spaces. J. Comput. Appl. Math. 228, 458-465 (2009)

27. Petrusel, A, Yao, JC: Viscosity approximation to common fixed points of families of nonexpansive mappings with generalized contractions mappings. Nonlinear Anal. TMA 69, 1100-1111 (2008)

28. Lau, ATM, Shioji, N, Takahashi, W: Existence of nonexpansive retractions for amenable semigroups of nonexpansive mappings and nonlinear ergodic theorems in Banach spaces. J. Funct. Anal. 161, $62-75$ (1999)

29. Chen, R, Song, Y: Convergence to common fixed point of nonexpansive semigroups. J. Comput. Appl. Math. 200 566-575 (2007)

30. Cianciaruso, F, Marino, G, Muglia, L: Iterative methods for equilibrium and fixed point problems for nonexpansive semigroups in Hilbert spaces. J. Optim. Theory Appl. 146, 491-509 (2010)

31. Chen, $\mathrm{R}, \mathrm{He}, \mathrm{H}$ : Viscosity approximation of common fixed points of nonexpansive semigroups in Banach space. Appl. Math. Lett. 20, 751-757 (2007)

32. Zegeye, $\mathrm{H}$, Shahzad, N: Strong convergence theorems for a finite family of nonexpansive mappings and semigroups via the hybrid method. Nonlinear Anal. TMA 72, 325-329 (2010)

33. Buong, N: Strong convergence theorem for nonexpansive semigroups in Hilbert space. Nonlinear Anal. TMA 72, 4534-4540 (2010)

34. Lau, AT, Takahashi, W: Fixed point properties for semigroup of nonexpansive mappings on Fréchet spaces. Nonlinear Anal. TMA 70, 3837-3841 (2009)

35. Lau, ATM, Nishiura, K, Takahashi, W: Nonlinear ergodic theorems for semigroups of nonexpansive mappings and left ideals. Nonlinear Anal. TMA 26, 1411-1427 (1996)

36. Lau, ATM: Semigroup of nonexpansive mappings on a Hilbert space. J. Math. Anal. Appl. 105, 514-522 (1985)

37. Lau, ATM, Miyake, H, Takahashi, W: Approximation of fixed points for amenable semigroups of nonexpansive mappings in Banach spaces. Nonlinear Anal. TMA 67, 1211-1225 (2007)

38. Lau, ATM, Takahashi, W: Invariant means and semigroups of nonexpansive mappings on uniformly convex Banach spaces. J. Math. Anal. Appl. 153, 497-505 (1990)

39. Lau, ATM, Takahashi, W: Invariant submeans and semigroups of nonexpansive mappings on Banach spaces with normal structure. J. Funct. Anal. 142, 79-88 (1996)

40. Lau, ATM, Zhang, Y: Fixed point properties of semigroups of non-expansive mappings. J. Funct. Anal. 254, 2534-2554 (2008)

41. Katchang, P, Kumam, P: An iterative algorithm for common fixed points for nonexpansive semigroups and strictly pseudo-contractive mappings with optimization problems. J. Glob. Optim. (2012). doi:10.1007/s10898-012-9927-y

42. Kumam, P, Wattanawitoon, K: A general composite explicit iterative scheme of fixed point solutions of variational inequalities for nonexpansive semigroups. Math. Comput. Model. 53, 998-1006 (2011)

43. Sunthrayuth, P, Kumam, P: A general iterative algorithm for the solution of variational inequalities for a nonexpansive semigroup in Banach spaces. J. Nonlinear Anal. Optim. 1, 139-150 (2010)

44. Chang, SS, Cho, YJ, Joseph Lee, HW, Chan, CK: Strong convergence theorems for Lipschitzian demi-contraction semigroups in Banach spaces. Fixed Point Theory Appl. 2011, Article ID 583423 (2011)

45. Cho, YJ, Ćirić, LB, Wang, SH: Convergence theorems for nonexpansive semigroups in CAT(0) spaces. Nonlinear Anal. 74, 6050-6059 (2011)

46. Yao, Y, Cho, YJ, Liou, YC: Hierarchical convergence of an implicit double-net algorithm for nonexpansive semigroups and variational inequality problems. Fixed Point Theory Appl. 2011, Article ID 101 (2011) 